\title{
When Wellens' syndrome meets Brugada phenocopy
}

\author{
Syed HR Naqvi ${ }^{1}$ and Han Naung Tun ${ }^{2}$ \\ ${ }^{1} \mathrm{CPE}$ Institute of Cardiology, Multan, Punjab, Pakistan \\ ${ }^{2}$ Clinical and Research Working Groups, European Society of Cardiology, Sophia Antipolis, France
}

\begin{abstract}
Wellens' syndrome, or "Widow Maker", is referred to as a pre-infarction syndrome with non-classical ischemic electrocardiographic (ECG) changes and unremarkable cardiac biomarkers. This syndrome shouldn't be missed by the clinicians as delay in urgent coronary intervention can result in anterior wall myocardial infarction (MI), arrhythmias, left ventricular dysfunction and death. It can be predicted by patient's history and ECG changes. Thus, physicians and health care providers should be familiar with the electrocardiographic manifestations about Wellens' Syndrome in clinical care. Here, we are describing a case of middle aged male patient presented with history of typical chest pain with slightly elevated blood pressure (BP) with an initial ECG showing Brugada pattern, patient was admitted and started with antiplatelets, anti-ischemics and anticoagulants followed by serial ECGs but after 3 hours ECG done showed wellenoid pattern for which he underwent a coronary angiography and it came out to be severe lesion in proximal left anterior descending (LAD) artery.
\end{abstract}

\section{Introduction}

Wellens' syndrome also known as LAD coronary T-wave syndrome characterized by history of anginal chest pain, characteristic ECG T-wave changes, without significant ST segment elevation, without $\mathrm{Q}$ waves and normal precordial R-wave progression with normal or minimally raised cardiac markers. Recognition of ECG within time is of greatest importance because this syndrome represents a pre-infarction stage of coronary artery disease that can progress to a lamentable anterior wall myocardial infarction (MI) [1].

Whereas, Brugada syndrome is an inherited cardiac disease without structural abnormalities can arise as a result of accelerated inactivation of Na channels and predominance of transient outward K current (Ito) to initiate a voltage gradient in the right ventricular layers triggering ventricular tachycardia/ventricular fibrillation possibly through a phase 2 re-entrant mechanism. The Brugada electrocardiographic (ECG) pattern, can be dynamic being recorded in upper precordial leads, is basically the hallmark of Brugada syndrome. The differential diagnosis could include Brugada-like pattern in the right precordial leads, especially athletes, right bundle-branch block, arrhythmogenic right ventricular dysplasia or cardiomyopathy and pectus excavatum [2].

Here it is important to mention the concept of Brugada phenocopies that ECG patterns are of characteristic Brugada pattern that may appear and disappear in relation with multiple causes but are not related with Brugada syndrome. The case which we're discussing here includes the clinical criteria and implications of Wellens syndrome and Brugada phenocopy [3].

\section{Case report}

A 53-year-old male with past medical history of treated pulmonary tuberculosis, presented in the hospital with severe chest pain for one hour, started while he was working in his garden. The pain radiated to both arms associated with nausea and sweating. He denied use of illicit substances, drugs, smoking and alcohol. He had no family history of coronary artery disease. Patient was pain free when he reached the hospital emergency.

Glasgow coma scale (GCS) was 15/15, blood pressure was $150 / 90$ $\mathrm{mmHg}$, heart rate was $68 \mathrm{beat} / \mathrm{min}$, respiratory rate was 18 breath/ min, temperature was $98 \mathrm{~F}$, and jugular venous pressure (JVP) was not raised. Rest of the physical examination was unremarkable. Cardiac examination revealed normal heart sounds with no clicks, gallop or murmurs. Chest, abdominal and neurologic examination was unremarkable. Written informed consent was taken and initial 12 lead ECG showed ST segment elevation in leads V1, V2, V3 with ST segment elevation similar to Brugada pattern (Type I), showed in figure 1.

Patient was admitted and given antiplatelet medication (Aspirin 300 $\mathrm{mg}$ and Clopidogrel $300 \mathrm{mg}$ stat). Anti-Ischemics and anticoagulants were started because the patient was started having chest pain on and off. Serial ECGs were done, and the ECG done after 10 hours was consistent with ischemia (wellenoid pattern). ECG showed ST elevation in lead V3 and V4 with prominent Biphasic T wave consistent with ischemia (wellenoid pattern). There is also a prominent $\mathrm{R}$ wave with mild ST Elevation in two contiguous leads and biphasic $\mathrm{T}$ wave inversions suggestive of Wellens' syndrome (Figure 2).

All baseline labs were normal except Troponin I which was found to be mildly elevated. Chest $\mathrm{X}$ ray and echocardiography was normal. Patient underwent Coronary angiography which showed severe proximal LAD stenosis (Figure 3 ) and that Brugada pattern came out to be Brugada phenocopy seen in V1 and V2 .

${ }^{\star}$ Correspondence to: Han Naung Tun, Clinical and Research Working Groups, European Society of Cardiology, Sophia Antipolis, France, E-mail: annasxhan@ gmail.com

Key words: Wellens, Brugada, anterior wall MI, left anterior descending, ischemia, ECG changes, angiography

Received: May 15, 2019; Accepted: May 25, 2019; Published: May 28, 2019 


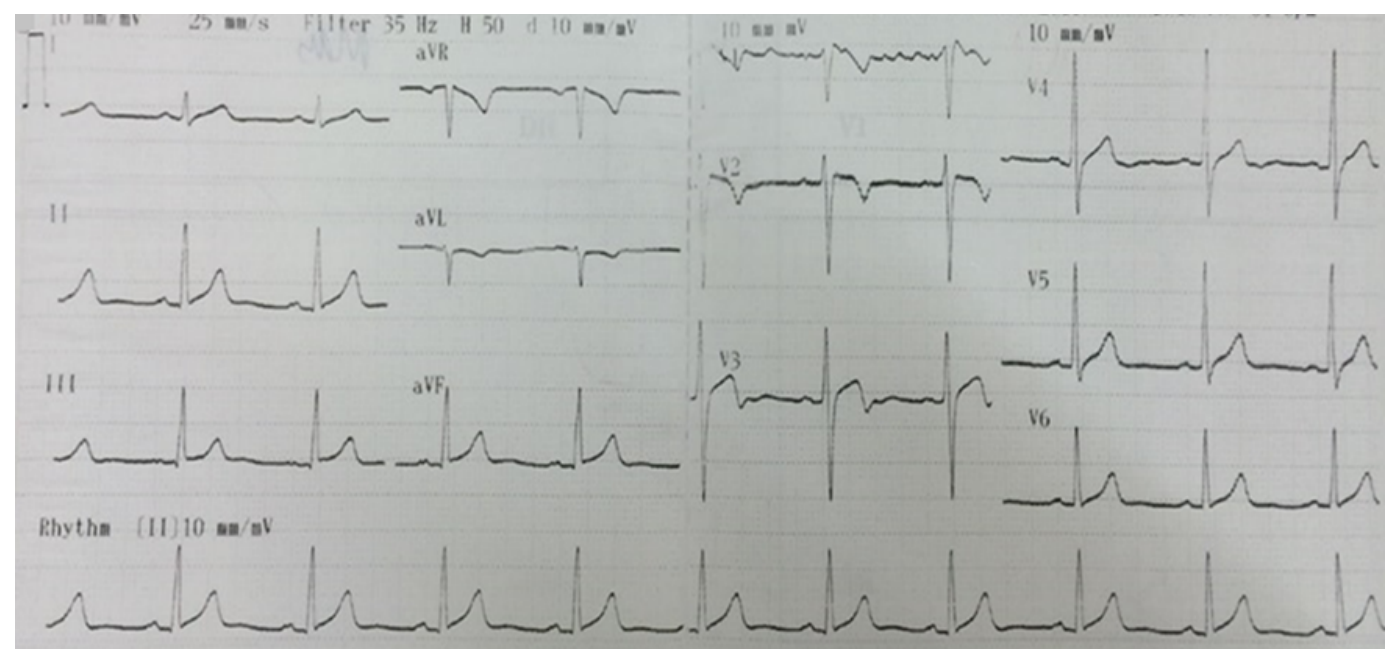

Figure 1. ECG on arrival in hospital emergency

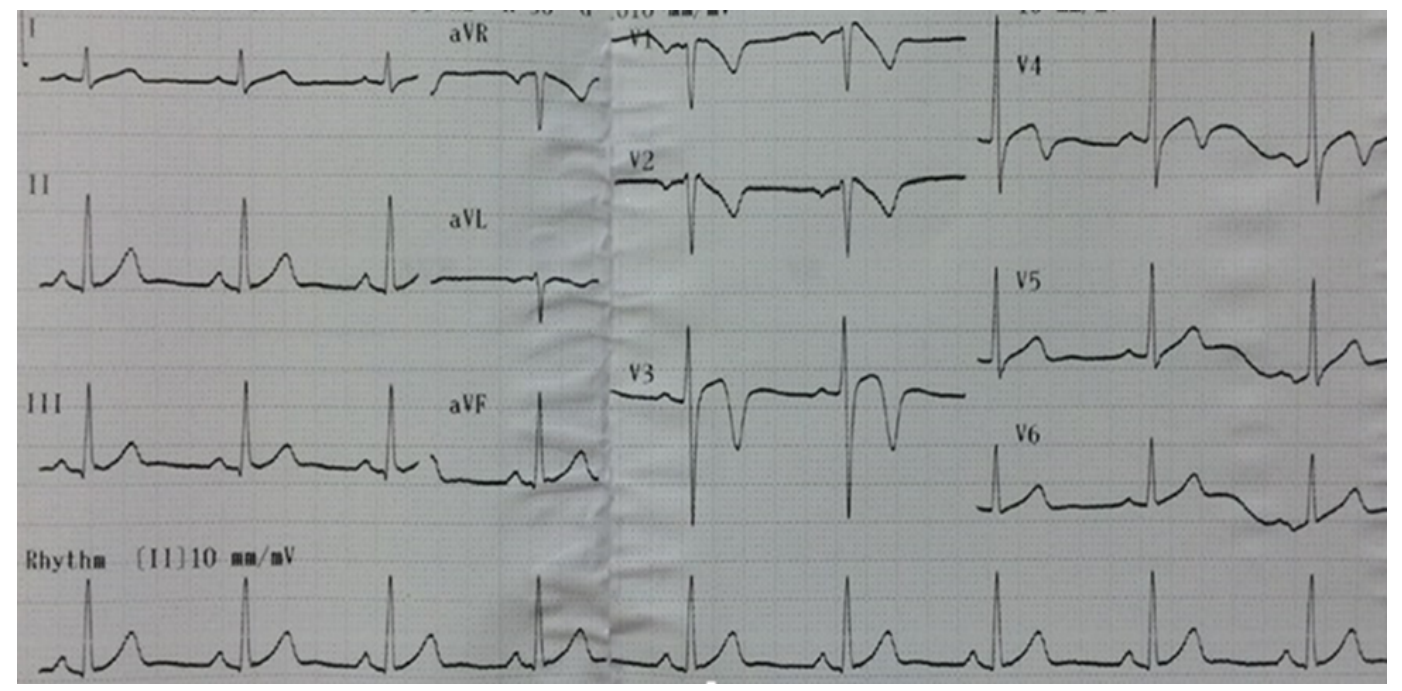

Figure 2. ECG done after 3 hours of initial ECG

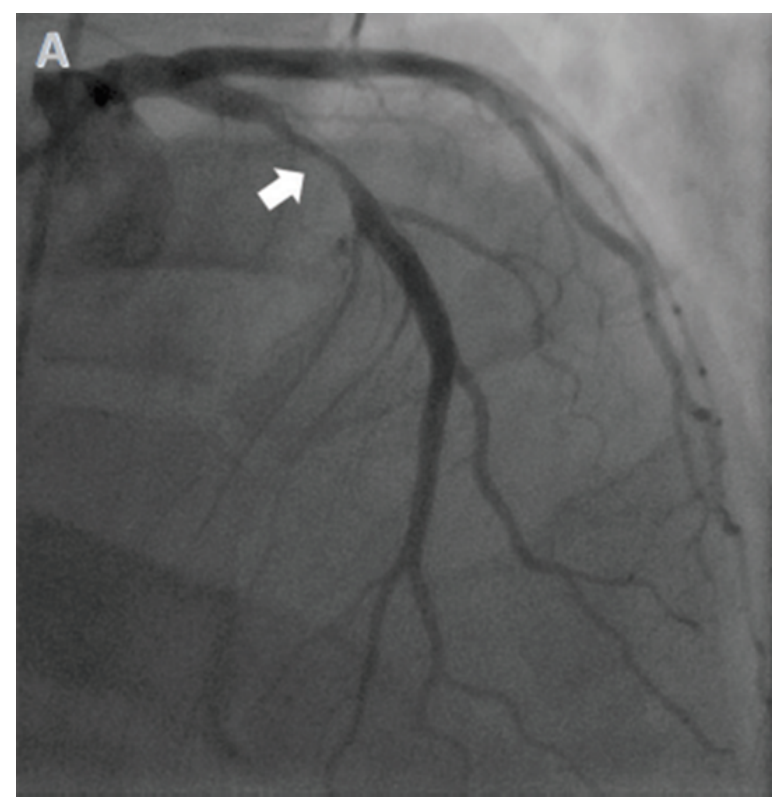

Figure 3. Coronary Angiogram showing severe disease in Proximal LAD 
PCI to LAD was done (Figure 4) with Drug eluting stent (DES), patient discharged on medications after 24 hours and telephonic follow-up was made after 1,2, and 4 weeks, patient is back to his usual routine life and rechecked ECG after stented to LAD was as shown in (Figure 5).

\section{Discussion}

Wellens' Syndrome, first described in 1982 by professor Hein J.J Wellens. It is rarely seen Wellens' syndrome and Brugada like ECG pattern come together in ischemic heart disease. This syndrome along with Brugada like ECG pattern has become one of the essential instant recognition abnormalities on ECG for emergency physicians. According to one data, this ECG pattern is strongly associated with a widow maker lesion - with $100 \%$ of 180 patients with the pattern having $>50 \%$ stenosis of LAD coronary artery $($ mean $=85 \%)$ with complete occlusion in almost $60 \%$ [4]. Criteria for recognizing this syndrome include a history of anginal chest pain, less than twice the upper limit of normal in cardiac biomarkers [5], ECG shows biphasic or deeply inverted $\mathrm{T}$ waves in the precordial leads obtained during a pain-free interval. Patient can present with both Type A Wellens', which comprises $25 \%$ of cases and has biphasic T waves in lead V2 and V3. The remaining $75 \%$ are Type B Wellens' syndrome, which is deeply inverted, symmetrical $\mathrm{T}$ waves predominantly in V2 and V3 [6].

The causes include atherosclerotic plaque, increase in cardiac demand, coronary artery vasospasm and hypoxia. The risk factors are same as those for coronary artery disease including diabetes, hypertension, dyslipidemia, obesity, sedentary lifestyle, familial history, and smoking. The exact mechanism is not known but usually it is caused by the rupture of an atherosclerotic plaque leading to LAD occlusion, followed by clot lysis or other disruption of the occlusion before complete MI has taken place. Some consider that coronary artery spasm and stunned myocardium can cause it. While some studies show that it is caused by frequent transmural ischemia-reperfusion leading to myocardial oedema [7].

Patients with this syndrome usually present with symptoms same as for acute coronary syndrome (ACS) include chest pain, chest tightness or pressure-like, induced by physical activity and relieved by rest. This pain may sometimes typically radiate to neck, jaw, or shoulder. The differential for anterior T-wave inversion (TWI) also includes right bundle branch block (RBBB), hypertrophic cardiomyopathy (HCM), left ventricular hypertrophy (LVH), pulmonary embolism (PE) or central nervous system (CNS) injury (so-called "cerebral" T waves) [8].

On the other hand, Brugada Syndrome was first described in 1992 by the Brugada brothers, is an inherited cardiac disease without structural abnormalities which arise as a result of accelerated inactivation of sodium $(\mathrm{Na})$ channels and predominance of transient outward $\mathrm{K}$ current (Ito) to generate a voltage gradient in the right ventricular layers could trigger into arrhythmias like ventricular tachycardia or ventricular fibrillation possibly occur through a phase 2 re-entrant

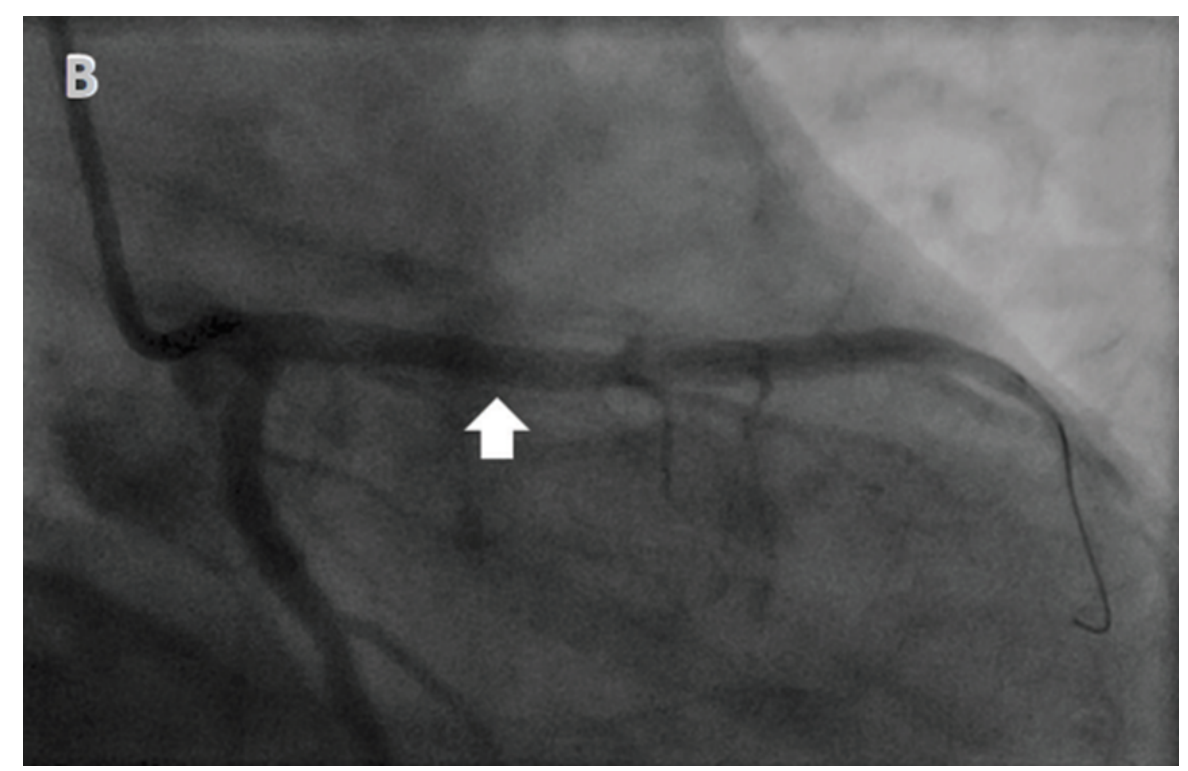

Figure 4. After placing stent in Proximal LAD

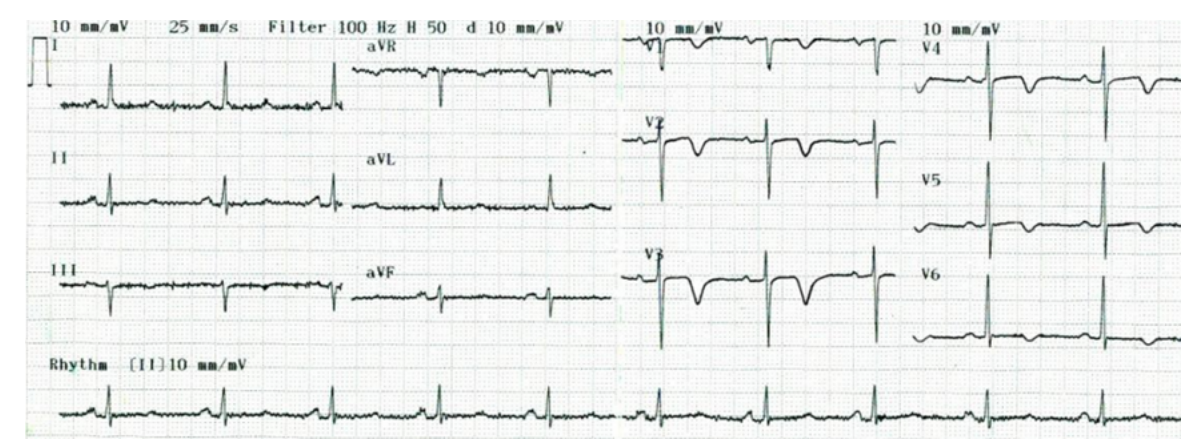

Figure 5. Rechecked ECG after stented to LAD 
mechanism as already discussed. The mean age of sudden death is 41 , with the diagnosing age ranging from 2 days to 84 years [2,9].

Diagnostic criteria for Brugada Syndrome includes Brugada type 1 Coved ST segment elevation $>2 \mathrm{~mm}$ in V1-V3 followed by T wave inversion whereas type 2 has $>2 \mathrm{~mm}$ of saddleback shaped ST elevation and Type 3 can be the morphology of either type 1 or type 2, but with $<2 \mathrm{~mm}$ of ST segment elevation [10]. The only proven therapy for Brugada Syndrome is an implantable cardioverter - defibrillator (ICD) device. Quinidine has also been introduced as an alternative therapy where ICDs are not available.

A similar case by Dr. Raimundo Barbosa Barros from Brazil has been extensively discussed [11], back in November 2010 that 56 years old male patient presented in emergency room claiming that in April 2010 , he was admitted with symptoms of unstable angina for which he underwent coronary angiography that revealed severe proximal lesion of the LAD and $90 \%$ of obstruction in the distal portion of the RCA. Back then, he underwent angioplasty with stent in the LAD; the RCA was not approached. On September 20th, 2010 routine myocardial perfusion imaging (MPI) was done (normal) but this time he presented after syncopal episode, preceded by palpitations and atypical chest discomfort for 4 hours with undocumented fever but he had no previous similar episode or positive family history. ECG done showed Type 1 Brugada Pattern. Serial measurement of CK-MB and troponin were also done normal. Advised for ICD implantation. So, this patient had CAD associated with Brugada-ECG pattern [11].

All in all, our case draws attention to the need for timely identification of Wellens' syndrome and the appropriate management to urgent coronary angiography for possible angioplasty and assessment for emergency coronary artery bypass grafting (CABG). According to data, $75 \%$ of non-vascularized patients will progress to acute anterior wall MIs within 1 week, if left untreated if proper recognition and time management for wellens' is impaired [12].

\section{Conclusion}

The purpose for presenting this case is that any patient with Brugada Pattern ECG can have underlying Wellens' syndrome. Coronary angiogram should be performed to rule out the coronary artery disease.
Ischemic changes can be confused with Brugada pattern. History and recognition of the ECG findings of both Type 1 and 2 Wellens' syndrome is important for proper management and emergent intervention to avoid large anterior wall MI. Hence the definitive treatment for wellens syndrome typically involves cardiac catheterization with percutaneous coronary intervention (PCI) to relieve the occlusion.

\section{References}

1. De Zwaan C, Bär FW, Wellens HJ (1982) Characteristic electrocardiographic pattern indicating a critical stenosis high in left anterior descending coronary artery in patients admitted because of impending myocardial infarction. Am Heart $J$ 103: 730-736. [Crossref]

2. Bayés de Luna A, Brugada J, Baranchuk A, Borggrefe M, Breithardt G, et al. (2012) Current electrocardiographic criteria for diagnosis of Brugada pattern: a consensus report. J Electrocardiol 45: 433-442. [Crossref]

3. Anselm DD, Evans JM, Baranchuk A. Brugada phenocopy: A new electrocardiogram phenomenon. World J Cardiol 6: 81. [Crossref]

4. Cadogan M (2019) What is Wellens' Syndrome? Available from: https:// lifeinthefastlane.com/what-is-wellens-syndrome/.

5. Hollar L, Hartness O, Doering T (2015) Recognizing Wellens' syndrome, a warning sign of critical proximal LAD artery stenosis and impending anterior myocardial infarction. J Community Hosp Intern Med Perspect 5: 29384. [Crossref]

6. Rhinehardt J, Brady WJ, Perron AD, Mattu A (2002) Electrocardiographic manifestations of Wellens' syndrome. Am J Emerg Med 20: 638-643. [Crossref]

7. Miner B, Hart EH (2019) Wellens syndrome. StatPearls. [Crossref]

8. Hayden GE, Brady WJ, Perron AD, Somers MP, Mattu A (2002) Electrocardiographic T-wave inversion: differential diagnosis in the chest pain patient. Am J Emerg Med 20: 252-262. [Crossref]

9. Antzelevitch C, Brugada P, Brugada J, Brugada R, Towbin JA, et al. (2003) Brugada syndrome: 1992-2002: a historical perspective. J Am Coll Cardiol 41: 1665-1671. [Crossref]

10. Antzelevitch C, Brugada P, Borggrefe M, Brugada J, Brugada R, et al. (2005) Brugada syndrome: report of the second consensus conference: endorsed by the Heart Rhythm Society and the European Heart Rhythm Association. Circulation 111: 659-670. [Crossref]

11. Barbosa-Barros R (2017) Brugada syndrome associated to Myocardial ischemia Available from: http://fiaiweb.com/wp-content/uploads/2017/09/Brugada-syndromeassociated-to-Myocardial-Ischemia2.pdf.

12. Mead NE, O'Keefe KP (2009) Wellen's syndrome: An ominous EKG pattern. J Emerg Trauma Shock 2: 206-208. [Crossref]

Copyright: (C2019 Naqvi SHR. This is an open-access article distributed under the terms of the Creative Commons Attribution License, which permits unrestricted use, distribution, and reproduction in any medium, provided the original author and source are credited. 